\title{
2 Zusammenarbeit und Kooperation in der Homecare-Versorgung
}

Die Homecare-Versorgung ist durch einen hohen Grad an Spezialisierung geprägt. Je höher jedoch der Grad der Spezialisierung einzelner Berufsgruppen, desto mehr wird dadurch die Versorgungslandschaft fragmentiert. Hinzu kommt, dass neben den versorgenden Leistungserbringern (Arzt, Klinik, Rehabilitationseinrichtung, ambulante Pflege und Pflegeheim) auch pflegende Angehörige sowie Kostenträger beteiligt sind. Es entstehen Schnittstellen zwischen allen Beteiligten, die unterschiedlichen Regelungsbereichen und Versorgungslogiken unterliegen (SVR, 2007).

Die Herausforderung liegt darin, die Vielzahl der im Versorgungsprozess Mitwirkenden zu koordinieren. An den Schnittstellen kommt es immer wieder zu Kommunikations- und Koordinationsproblemen, die zu Versorgungsbrüchen für den Patienten führen. In einer solch segmentierten Versorgungslandschaft braucht es daher generalistisch ausgerichtete Berufsgruppen, die eine Lotsenfunktion im Sinne eines Case-Managers für den Patienten, aber auch für die betroffenen Leistungserbringer erfüllen (SVR, 2007). HomecareUnternehmen haben das Potential, den Versorgungsprozess des Patienten als Case-Manager zu koordinieren und die Aktivitäten aller Beteiligten zusammenzuführen und aufeinander abzustimmen. Die professionelle Umsetzung dessen bedarf jedoch einer genaueren Analyse der Ausgestaltung und Bedingungen der Zusammenarbeit. Aufgabenbereiche und Verantwortlichkeiten sind haftungssicher zu definieren. 


\subsection{Zusammenarbeit im Gesundheitswesen}

Mit der Einführung verschiedener neuer Versorgungsformen, wie beispielsweise der Integrierten Versorgung, den Disease Management Programmen oder der Entwicklung Medizinischer Versorgungszentren versucht der Gesetzgeber bereits seit mehreren Jahren strukturelle Vielfalt im Gesundheitswesen zu stärken. In der Annahme, dass sich die Gesundheitsversorgung auch künftig weiter in Richtung eines kooperativen Systems entwickeln wird, das gekennzeichnet ist durch eine verstärkte Zusammenarbeit in Teams, einer Orientierung an Prozessen und Qualität sowie einer Ausrichtung auf den Patienten, müssen sich die Prozesse der Arbeitsteilung zwischen den Gesundheitsberufen verändern, Kooperationsbeziehungen gestaltet und Arbeitsaufgaben entsprechend neu definiert werden (SVR, 2007).

Professionelle Zusammenarbeit setzt eine eindeutige Definition und Abgrenzung der jeweiligen Aufgabenbereiche voraus (vgl. Abbildung 12). Innerhalb einer Berufsgruppe kann das Aufgabenspektrum im Sinne einer Diversifikation oder Spezialisierung neu definiert werden. Wenn das Aufgabengebiet einer Arztgruppe beispielsweise durch modernere Technologien oder Behandlungsverfahren ausgeweitet wird, spricht man von einer Diversifikation. Im Gegensatz dazu zielt eine Spezialisierung auf die Konzentration und damit Verdichtung eines Teilgebietes des bisherigen Aufgabenspektrums ab. Die Spezialisierung erfordert in der Regel zusätzliche Kenntnisse oder Fertigkeiten, die über spezielle Schulungen erworben werden.

Unter Substitution versteht sich die vollständige Übernahme bestimmter Tätigkeitsfelder einer Berufsgruppe durch eine andere. Aufgaben können dabei horizontal bei gleichwertigem Ausbildungsniveau, wie etwa zwischen Facharztgruppen, oder vertikal bei ungleichem Ausbildungsgrad, z.B. an nicht-ärztliches Personal verschoben werden. Im Vergleich dazu beschränkt sich eine Delegation von Aufgaben auf eine temporär angeordnete Übertragung von beruflichen Tätigkeiten (SVR, 2007).

Neudefinition des Aufgabenspektrums
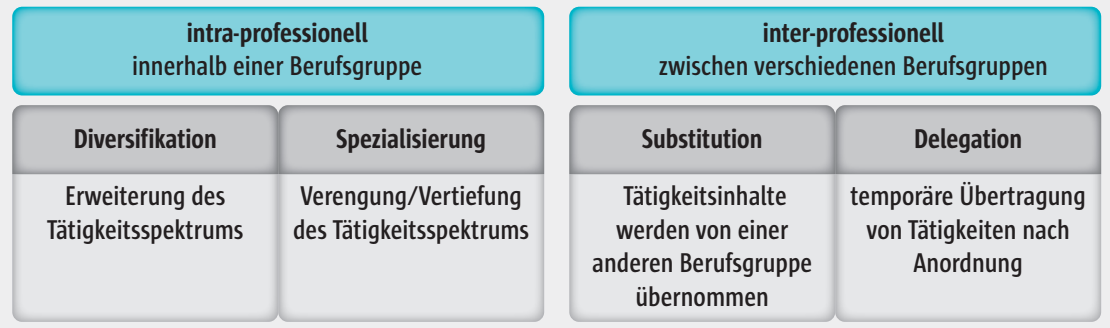

Abb. 12 Neudefinition des Aufgabenspektrums im Gesundheitswesen (eigene Darstellung in Anlehnung an SVR, 2007) 
Substitution und Delegation setzen Schnittmengen zwischen den Kompetenzen zweier Berufsgruppen voraus. Unterliegen hingegen einzelne Tätigkeiten einem Vorbehalt, dürfen diese ausschließlich von der ihr zugewiesenen Berufsgruppe ausgeführt werden. Weitreichende Tätigkeitsvorbehalte sind jedoch eher kontraproduktiv für eine flexible Zusammenarbeit. Bei einer Neudefinition der Aufgabenfelder bietet es sich daher an, die Kernkompetenzen einer Berufsgruppe zu hinterfragen und zu diskutieren. Sie dienen der Identitätsbildung für die Profession und legen die Verantwortlichkeiten fest. Aufgabengebiete, die hingegen von mehreren Gesundheitsberufen abgedeckt werden können - wie z.B. Case-Management oder Prävention - können als sogenannte Poolkompetenzen beschrieben werden. Diese meist interdisziplinären Aufgaben sollten mit Blick auf ein kooperatives und flexibles Versorgungssystem zwingend nicht nur einer Berufsgruppe zugeteilt werden.

\subsection{Erfahrungen Beteiligter mit interprofessioneller Zusammenarbeit}

Die Erfahrungen und Ansichten von Ärzten, Praxisassistenten, Pflegekräften oder auch Patienten und Angehörigen hinsichtlich der interprofessionellen Zusammenarbeit in der Homecare-Versorgung, sind zahlreich untersucht.

Sowohl Ärzte als auch Pflegekräfte beurteilen interprofessionelle Zusammenarbeit als grundlegend positiv und wertvoll für die eigene Arbeit sowie für die Patienten (Bussche et al., 2013). Trotz der wahrgenommenen Möglichkeiten besteht jedoch erhebliches Verbesserungspotential hinsichtlich einer gezielten und abgestimmten interprofessionellen Zusammenarbeit zwischen ambulanten Pflegediensten, behandelnden Ärzten und weiteren Beteiligten. So bewerteten Hausärzte in einer Studie von Osterbrink et al. (2013) die Qualität der Zusammenarbeit mit Pflegenden der ambulanten Pflegedienste mit einer Note von 2,7 und die Zusammenarbeit mit Mitarbeitern des Palliativnetzwerks mit 1,7.

Trotz der Vielzahl der Studien ergibt sich ein recht einheitliches Bild über die Erfolgsfaktoren einer funktionierenden interprofessionellen Zusammenarbeit und damit auch Delegation. Ein aktueller Review von Niezen und Mathijssen (2014) aus den Niederlanden identifiziert vier Kategorien für fördernde und hemmende Faktoren:

1. Wissen und Fähigkeiten

2. professionelle Grenzen

3. organisatorische Umgebung

4. institutionelle Umgebung

Als wichtigster Faktor für eine funktionierende interprofessionelle Zusammenarbeit wird insbesondere von Ärzten die Fachkompetenz des beteiligten 
Personals genannt (Niezen \& Mathijssen, 2014; Pype et al., 2013). Gleichzeitig setzt eine erfolgreiche Zusammenarbeit gegenseitiges Vertrauen und eine eindeutige Definition der Rollen, Aufgaben und Verantwortung voraus. Häufig sind die Beziehungen jedoch von einem hierarchischen Gefälle geprägt. Kooperation und Teamwork sollten in die Ausbildung von Ärzten und Pflegekräften integriert werden, um die Arbeitsweisen und Denklogiken der jeweils anderen Berufsgruppen zu vermitteln.

Pflegende wirken häufig als Vermittler zwischen dem Hausarzt und dem Patienten. Auch wenn die Patienten den Hausarzt als primären Ansprechpartner in Bezug auf die Versorgung sehen, sind die Pflegekräfte für den Patienten der direkte Ansprechpartner, da sie wöchentlich bis mehrmals täglich mit ihm in Kontakt und somit regelmäßig über seinen Zustand informiert sind. Etwa die Hälfte der Pflegekräfte gibt an, dass sie bei der Versorgung des Patienten einen Handlungsspielraum haben (Osterbrink et al., 2013). Umso wichtiger ist es, dass Klarheit über die Ziele der Versorgung und die Verantwortungsbereiche der Einzelnen, z.B. im Rahmen von Teamvereinbarungen besteht (Pype et al., 2013). Close et al. (2013) verglichen die Erfahrungen von herzkranken Patienten, die auf Grundlage eines speziellen Managementplans von „Herzschwäche“-Krankenschwestern versorgt wurden mit denen von Patienten der Regelversorgung. Auch hier zeigte sich, dass die Verantwortungsbereiche in der Regelversorgung nicht eindeutig geregelt sind. Ärzte und Pflegende gingen oftmals davon aus, dass die Entscheidung, die Versorgung und die Koordination Aufgabe des jeweils anderen sei.

Auch die organisatorische Umgebung kann eine erfolgreiche interprofessionelle Zusammenarbeit und Delegation wesentlich fördern, indem sie insbesondere eine funktionierende Kommunikation unterstützt, z.B. durch eine gemeinsame Dokumentation und interdisziplinäre Fallbesprechungen. Neben erheblichen Unstimmigkeiten beim Übergang vom stationären in den ambulanten Bereich hindern ähnliche Barrieren auch die interprofessionellen Zusammenarbeit zwischen Hausärzten und Pflegediensten (Bussche et al., 2013; Götze et al., 2010). Obwohl die Gestaltung der Kommunikation für die Zusammenarbeit zwischen den Beteiligten unerlässlich ist, wird diese Kommunikation meist individuell geregelt. Nur knapp die Hälfte der Hausärzte nutzt eine gemeinsame Dokumentation, obwohl mehr als die Hälfte beider Berufsgruppen eine für die Zusammenarbeit unzureichende Dokumentation bemängelten. Unterschiedliche Informationsstände zwischen Hausärzten und Pflegenden führen häufig zu Missverständnissen (Osterbrink et al., 2013).

Darüber hinaus haben die institutionellen Rahmenbedingungen entscheidenden Einfluss auf die interprofessionelle Zusammenarbeit. Darunter fällt auch die Gesetzgebung, die eine Barriere für eine Neuverteilung von Verantwortungsbereichen insbesondere bei nicht eindeutig geklärten Haftungsfragen darstellt (Niezen \& Mathijssen, 2014). 


\begin{abstract}
Exkurs - neue Ansätze der interdisziplinären Homecare-Versorgung
International werden im Zuge des erwarteten Personalmangels in der Pflege auch alternative Ansätze der interdisziplinären Zusammenarbeit im HomecareBereich diskutiert und erprobt. So werden in den USA beispielsweise Physiotherapeuten in die Wundversorgungsteams integriert (Abeln \& Pitassi 2012). Bei einer entsprechenden Qualifikation kann der Physiotherapeut Wundpatienten dabei unterstützen, Druckentlastungskissen richtig zu positionieren sowie über ein Fitnessprogramm für zu Hause Zirkulation, Kraft, Mobilität sowie Gleichgewicht zu verbessern und Ödeme vorzubeugen. Die Wundheilung kann damit entscheidend begünstigt werden. Aber auch hier stellten sich als entscheidende Erfolgsfaktoren für die interdisziplinäre Wundversorgung die Qualifikation des Personals sowie Teamwork und effektive Kommunikation heraus.
\end{abstract}

\title{
2.3 Die Rolle des Hausarztes in der Homecare-Versorgung
}

Historisch gewachsen, kommt dem Arzt im deutschen Gesundheitswesen eine überaus zentrale und starke Position in der Patientenversorgung zu (SVR, 2007). Mit dem Gesetz über das Kassenarztrecht 1955 erhielt die Profession des Arztes durch die Schaffung der Kassenärztlichen Vereinigung als Körperschaft des öffentlichen Rechts eine institutionalisierte Interessenvertretung mit Selbstverwaltungsrecht. Über die zeitgleiche Bildung des Bundesausschuss der Ärzte und Krankenkassen erhielten die Ärzte zudem die Befugnis, gemeinschaftlich mit den Kassen Richtlinien über die Verordnung von Arznei- und Heilmitteln zu erstellen. Die Heilmittelleistungserbringer sind demnach von der Definition des Umfangs und Inhalts einer in der GKV ausreichend und zweckmäßigen Leistung ausgenommen. Ebenso erfolgt eine Planung des Bedarfs an Leistungserbringern bislang lediglich für Vertragsärzte (neben der Krankenhausplanung). Mit der Definition des ärztlichen Verschreibungsmonopols im SGB V wird den Ärzten auch die zentrale Position im Versorgungsgeschehen eingeräumt. Hilfeleistungen anderer Personen dürfen demnach nur erbracht werden, wenn sie vom Arzt angeordnet wurden und von ihm verantwortet werden ( $\$ 15$ Abs. 1 SGB V).

Homecare steht für eine ärztliche Behandlung eines Patienten im außerklinischen Bereich. Nach der Klinikentlassung muss die dort begonnene Behandlung vom niedergelassenen Arzt neu veranlasst und verordnet werden, um die Weiterbehandlungen und den therapeutischen Erfolg zu gewährleisten. Somit unterliegen die meisten Homecare-Versorgungsleistungen dem Arztvorbehalt bzw. der ärztlichen Verordnung, wodurch der Arzt auch hier eine Schlüsselfunktion einnimmt (Schanz, 2009).

Nicht-ärztliche Gesundheitsberufe stehen dem weit hintenan, weder wird ihr Bedarf geplant, noch haben sie das Anrecht auf eine institutionalisierte Interessensvertretung. In anderen Ländern, insbesondere Großbritannien 
oder den USA, sind die nicht-ärztlichen Professionen hingegen wesentlich stärker organisiert. Auch bei der Umsetzung von neuen Versorgungsformen sind nicht-ärztliche Gesundheitsberufe zumeist unterrepräsentiert. Die dezentrale Vertragsbildung im Rahmen neuer Versorgungsformen berücksichtigt zumeist primär medizinische Inhalte mit Krankenkassen und ärztlichen Leistungserbringern als Hauptvertragspartner. Die Zusammenarbeit zwischen den Gesundheitsberufen ist selten expliziter Vertragsbestandteil und selbst dort, wo nicht-ärztliche Berufe eingebunden sind, verbleiben diese meist in ihren traditionellen Rollen und Abhängigkeiten (SVR, 2007).

\subsection{Homecare und ärztliche Delegation}

Per Gesetz ist der Arzt dazu verpflichtet, die ärztliche Behandlung als Dienstleistung persönlich zur erbringen. Er kann sich jedoch teilweise durch Assistenten zuarbeiten lassen und bestimmte Aufgaben an ausreichend qualifizierte Mitarbeiter delegieren (SVR, 2007). Dabei bleibt der Arzt jedoch immer in der Gesamtverantwortung, sowohl für die Diagnose als auch für die indizierte Therapie. Außerdem muss er sich von der Qualifikation und Kompetenz des Delegationsempfängers strukturell und im Behandlungsprozess persönlich überzeugen.

In der derzeitigen Homecare-Praxis ist es häufig der Fall, dass die Klinik einen Patienten mit bestehendem Homecare-Versorgungsbedarf bei der Entlassung meist direkt dem Homecare-Lieferanten meldet. Dieser übernimmt die Versorgung des Patienten, indem er auch über die Indikationsstellung und die Produktwahl entscheidet und die beteiligten Pflegekräfte in die Handhabung der Produkte einweist. Der Hausarzt, der normalerweise den Behandlungsauftrag für den Patienten nach der Entlassung inne hat, wird häufig erst und meist ausschließlich zur Rezeptausstellung eingebunden. Vom Prinzip her ist das eine klassische Delegationsbeziehung des Homecare-Lieferanten gegenüber dem behandelnden Arzt. Nicht-ärztliches Personal erbringt ärztliche Leistungen, wobei die Gesamtverantwortung der Behandlung beim Arzt bleibt (Neumann et al., 2014). Allerdings erfolgt diese Delegation bislang wenig zielgerichtet, bewusst oder koordiniert und birgt daher Risiken für alle Beteiligten - sowohl Patienten als auch Hausärzte.

\subsubsection{Nationale und internationale Entwicklung der Zusammenarbeit im Gesundheitswesen}

Internationale Ansätze zur Delegation und Substitution, insbesondere aus den angloamerikanischen Ländern sowie den Niederlanden und Skandinavien, können für Deutschland eine Vorbildfunktion einnehmen (Dreier et al., 2012). In Ländern wie Australien, Neuseeland, Kanada, UK, Schweiz und Schweden werden die Fachkräfte akademisch auf Bachelor- oder gar Master- 
niveau ausgebildet und praxiserfahrene Krankenpflegekräfte systematisch weitergebildet (Neumann et al., 2014). Sogenannte Nurse Practitioners, die den Erstkontakt zu den Patienten verantworten und den weiteren Behandlungsprozess steuern und organisieren, sind bereits fest etabliert (Dreier et al., 2012).

In Neuseeland können Krankenpflegekräfte mit einem Masterabschluss z.B. Verschreibungen von Arznei-, Hilfs- und Heilmitteln vornehmen. In England und den Niederlanden werden die Krankenpflegekräfte innerhalb von Hausarztpraxen eingesetzt, um die Hausärzte zu unterstützen und von bürokratischem Aufwand zu entlasten. In den Niederlanden unterstützen diese ebenfalls die ambulante Versorgung außerhalb der üblichen Sprechzeiten, indem sie die weniger komplexen Fälle versorgen und somit die ärztliche Arbeitskraft sogar substituieren. Krankenpflegekräfte leiten in Kanada ambulante Behandlungszentren unter ärztlicher Überwachung. In Australien ist dies sogar ohne ärztliche Überwachung möglich. Hier behandeln die Pflegekräfte die Patienten anhand eines vorgegebenen Protokolls (Neumann et al., 2014).

In Deutschland forderte der Sachverständigenrat erstmals im Jahr 2007 eine Weiterentwicklung der Kooperation zwischen den Gesundheitsberufen (SVR, 2007). Der GKV Spitzenverband sieht in einer neuen Arbeitsteilung der Gesundheitsberufe insbesondere das Potential, die hausärztliche Versorgung in dünn besiedelten Gebieten zu stärken, und im Koalitionsvertrag 2013 formulieren CDU/CSU und SPD die Absicht, delegierte Aufgaben leistungsgerecht zu vergüten, um sie zur Regelleistung überführen zu können (CDU/CSU \& SPD, 2013). Seit Oktober 2013 gilt die Delegations-Vereinbarung im Rahmen des Bundesmantelvertrags, die regelt, welche ärztlichen Tätigkeiten delegiert werden dürfen und welche Anforderungen nicht-ärztliche Fachkräfte zu erfüllen haben (KBV \& GKV-Bund, 2013).

Trotz dieser Entwicklungen in den vergangenen Jahren hängt Deutschland dem internationalen Fortschritt im Bereich der Delegation weit hinterher (Neumann et al., 2014). Substitutionsvorhaben werden von der Ärzteschaft weitgehend abgelehnt, sodass es in diesem Bereich nur erste zurückhaltende Modellprojekte gibt. Etwas weiter fortgeschritten ist die Umsetzung von Delegationsvorhaben. Hier gibt es bereits eine Vielzahl an Modellprojekten, darunter AGnES und VerAH als die bekanntesten. Allerdings zeigt sich auch hier die praktische Umsetzung unter den Ärzten zurückhaltend. Aus dem Ärztemonitor 2014 geht hervor, dass 41\% der niedergelassenen Ärzte in Deutschland das Medikations- und Wundmanagement delegieren. Bis zu 18\% delegieren noch Hausbesuche. In 20\% der Arztpraxen sind für die Übernahme von delegierten Aufgaben weitergebildete Angestellte vorhanden (vgl. Abbildung 13). Lediglich 30\% der niedergelassenen Ärzte (39\% Hausärzte, 21\% Fachärzte, vgl. Abbildung 14) planen die Delegation an nicht-ärztliches Personal in Zukunft auszuweiten (infas, 2014). 


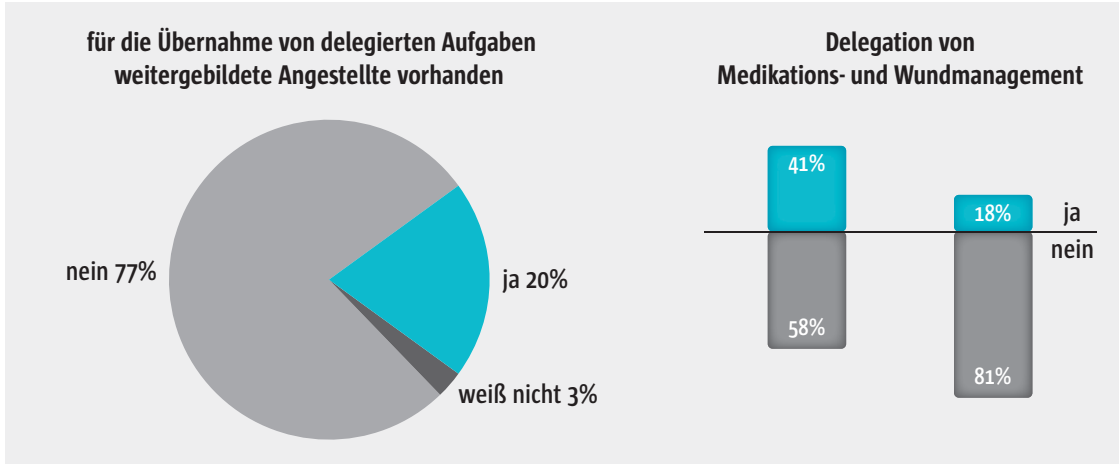

Abb. 13 Delegation in der Ärzteschaft 2014 (eigene Darstellung in Anlehnung an infas, 2014)

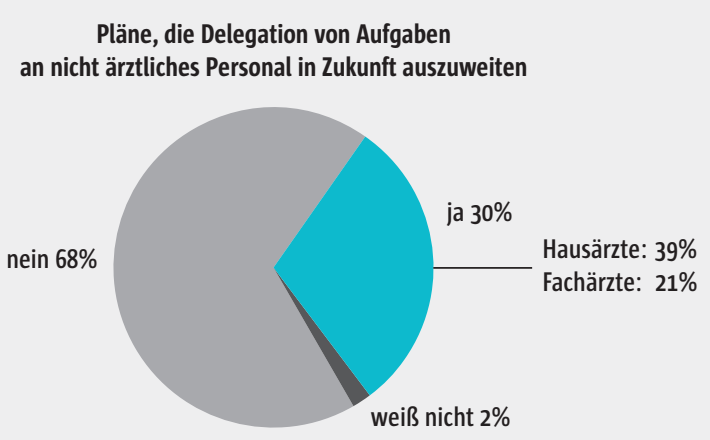

Abb. 14 Pläne der Ärzteschaft zur Ausweitung der Delegation (eigene Darstellung in Anlehnung an infas, 2014)

Das im Jahr 2015 in Kraft getretene Versorgungsstärkungsgesetz greift die weitere Flexibilisierung der vertragsärztlichen Versorgung und Entlastung durch Delegation erneut als einen Schwerpunkt auf. So beinhaltet das Gesetz Maßnahmen zum Ausbau des Einsatzes qualifizierter nicht-ärztlicher Gesundheitsberufe, die delegierte Leistungen erbringen. Insbesondere ist die Erbringung delegationsfähiger Leistungen nicht mehr länger nur auf unterversorgte Regionen beschränkt und soll bei der Weiterentwicklung des einheitlichen Bewertungsmaßstabes (EBM) berücksichtigt und angemessen bewertet werden. Das bestehende Potential für eine Delegation ärztlicher Leistungen soll durch das Versorgungsstärkungsgesetz möglichst voll ausgeschöpft werden. Auch die Umsetzung von Modellvorhaben zur Substitution soll vereinfacht und berufsrechtliche Regelungen hierzu angepasst werden (Deutscher Bundestag, 2015). Damit wird die politische Zielrichtung einer weiteren Forcierung der Arbeitsteilung und Kooperation zwischen den Gesundheitsberufen deutlich. Offen bleibt bislang, wie weit sich die betroffenen Akteure dieser Zielrichtung anpassen. Nach Untersuchungen des Ärz- 
temonitors plant die Mehrheit der befragten Ärzte keine Ausweitung der Delegation von ärztlichen Aufgaben (vgl. Abbildung 14, infas, 2014).

\subsubsection{Potentiale der Delegation}

Das größte Potential der Delegation wird darin gesehen, Beschäftigungsengpässe auf dem Arbeitsmarkt aufzufangen und die Arbeitsbelastung der delegierenden Berufsgruppe zu reduzieren (Neumann et al., 2014). Versorgungsprozesse können damit unter Stärkung des Teamgedankens flexibler gestaltet werden (SVR, 2007).

Berg et al. (2012) zeigten in diesem Zusammenhang beispielsweise, dass durch den Einsatz der Gemeindeschwester AGnES im Durchschnitt der sechs untersuchten Praxen 133 zusätzliche Patienten pro Quartal behandelt werden konnten. Ebenso konnte die Anzahl an Hausbesuchen durch Hausärzte nach der Einführung des AGnES-Konzepts signifikant gesenkt werden - darunter insbesondere die medizinisch dringenden Hausbesuche. Die Gesamtanzahl an Hausbesuchen ist durch die Delegation der Hausbesuche an AGnES-Kräfte auch nicht signifikant gestiegen.

In einer Studie von Dini, Sarganas, Heintze, und Braun (2012) nannten 70\% der befragten Ärzte die Zeitersparnis als wichtigen Nutzen der Delegation von Hausbesuchen an qualifizierte Assistenten, gefolgt von 48\%, die den Anstieg der eigenen Arbeitszufriedenheit schätzten. Ein Drittel der Ärzte vermutete auch einen Zufriedenheitsanstieg aufseiten der Praxisassistenten. Mit der Entlastung der einen Seite und dem Qualifikations- und Verantwortungszuwachs auf der anderen birgt eine Delegation das Potential, die Arbeitszufriedenheit beider Seiten zu erhöhen und zur Attraktivität des jeweiligen Berufsbildes beizutragen (SVR, 2007).

Nicht eindeutig beantwortet werden kann, ob das Mehr an Zeit, das für den Patienten zur Verfügung steht, auch zu einer gesteigerten Versorgungsqualität bzw. Patientenzufriedenheit führt. Möglicherweise hat der Patient gegenüber einer Fachkraft eine geringere Hemmschwelle, Informationen über sein Gesundheitsverhalten zu offenbaren, als gegenüber einem Arzt (Neumann et al., 2014). Allerdings wird häufig mit einer Skepsis der Patienten gegenüber der Delegation argumentiert. Nach Dini et al. (2012) befürchten $15 \%$ der Ärzte einen Vertrauensverlust zwischen ihnen und ihren Patienten. International hat sich jedoch gezeigt, dass die Akzeptanz der Patienten mit zunehmender Erfahrung steigt (Neumann et al., 2014). Mundiger et al. (2000) weisen für Großbritannien eine höhere Zufriedenheit der Patienten durch die Betreuung von Nurse Practitioners nach. Hingegen ist in der sechsmonatigen Studie nach Mundiger et al. (200o) in Amerika der Einfluss auf den Gesundheitsstatus sowie die Patientenzufriedenheit durch den Einsatz von Nurse Practitioners gleich geblieben. 
30\% der Hausärzte lehnten selbst bei entsprechender Vergütung die Delegation von Hausbesuchen ab (Dini et al., 2012). Die hauptsächlichen Barrieren liegen in der Ausgabensteigerung durch die Delegation und dem fehlenden Mehrwert für die Hausärzte. Der Großteil der Befragten findet es wichtig, dass das delegierte Personal ausschließlich in der eigenen Praxis angestellt ist und vor allem organisatorische und technische Fähigkeiten vorweist.

Die größte Hürde für die Verbreitung von Delegation liegt wohl in der offenen Frage der Finanzierung (Neumann et al., 2014). Insbesondere die Kosten der Qualifikation des Praxispersonals waren 34\% der befragten Ärzte in der Studie von Dini et al. (2012) zu hoch. 47\% der Hausärzte wären generell bereit, ihre Hausbesuche zu delegieren, wenn sie dafür entsprechend vergütet würden.

Sorge besteht auch hinsichtlich einer möglichen Leistungsausweitung, wenn die delegierten Leistungen überwiegend zusätzlich anstatt alternativ erbracht werden (Neumann et al., 2014; SVR, 2007). Davon hängt letztlich ab, inwieweit erwünschte Kosteneinsparungen eintreten. Akademisch ausgebildete Krankenpflegekräfte haben einerseits ein höheres Gehaltsniveau als nicht akademische, gleichzeitig bringen sie oftmals auch mehr Zeit für den Patienten auf. Dadurch führt Delegation selten zu den gewünschten Kosteneinsparungen, auch wenn Studienergebnisse oftmals auf eine ähnlich hohe Behandlungsqualität wie bei Ärzten hinweisen (Neumann et al., 2014).

Letztlich entstehen mit der Delegation weitere Schnittstellen in der Patientenversorgung, die mit einer Gefahr von Koordinations- und Kommunikationsfehlern und damit einer Gefährdung der Patientensicherheit einhergehen. Bei der Frage der Haftung besteht zumeist große Rechtsunsicherheit (SVR, 2007).

\subsubsection{Delegation von Homecare-Leistungen}

\section{Aktuelle rechtliche Situation in Deutschland}

Zwischen der Kassenärztlichen Bundesvereinigung und dem GKV-Spitzenverband gilt die Vereinbarung über die Delegation ärztlicher Leistungen an nicht-ärztliches Personal in der ambulanten vertragsärztlichen Versorgung (KBV \& GKV-Bund, 2013). Zuletzt wurde diese im Mai 2014 angepasst. Ihr zufolge entscheidet der Arzt aktiv, ob und an wen er eine Leistung delegiert. Er hat zu gewährleisten, dass er über eine schriftlich vereinbarte Weisungsbefugnis verfügt.

Darüber hinaus ist der Arzt in der Pflicht,

- die Eignung der Mitarbeiter für die delegierte Leistung zu prüfen (Auswahlpflicht),

- den Mitarbeiter zur selbstständigen Durchführung anzuleiten (Anleitungspflicht) und

- den Mitarbeiter regelmäßig zu überwachen (Überwachungspflicht). 
Dabei entscheidet die Qualifikation des Mitarbeiters über den Umfang der Anleitung und Überwachung.

Anamnese, Indikationsstellung, Untersuchung, Diagnosestellung, Aufklärung und Beratung des Patienten, Entscheidungen über die Behandlung sowie Durchführung invasiver Therapien und operative Eingriffe dürfen jedoch ausdrücklich nicht vom Arzt delegiert werden (\$2). Sie gelten als höchstpersönliche Aufgaben des Arztes und unterliegen dem Arztvorbehalt.

Darüber hinaus ist die Delegation ärztlich angeordneter Hilfeleistungen an nicht-ärztliche Praxisassistenten in der Häuslichkeit des Patienten oder in Alten- oder Pflegeheimen in einer weiteren Vereinbarung als Anlage zum Bundesmantelvertrag geregelt (KBV \& GKV-Bund, 2015). Die Ausführung und Abrechnung der delegierten Leistungen unterliegen der Genehmigungspflicht durch die Kassenärztliche Vereinigung (\$6).

Neben der Erfüllung der Qualifikationsvoraussetzungen muss die Praxisassistenz mit einer regelmäßigen Wochenarbeitszeit von mindestens 20 Stunden in der Arztpraxis angestellt sein. Die Behandlung und Betreuung von Patienten durch die Praxisassistenz außerhalb der Arztpraxis ist nur vorgesehen für über 65-jährige Patienten, die an einer schwerwiegenden chronischen oder akuten Erkrankung, die intensiver ärztlicher Betreuung bedarf, leiden und aufgrund ihres Gesundheitszustands nicht oder nur unter erschwerten Bedingungen die Praxis aufsuchen können.

Der behandelnde Arzt muss bei einer Delegation außerhalb der Arztpraxis den Patienten bereits bezüglich derselben Erkrankung gesehen und eingehend untersucht haben ( $\$ 5$ Absatz 2).

\section{Praktische Umsetzung der Delegation im Homecare-Bereich}

Die Versorgungspraxis sieht in der Regel so aus, dass eine Klinik die bevorstehende Entlassung eines Patienten mit Homecare-Versorgungsbedarf an den Lieferanten von Homecare-Produkten meldet. Die Krankenpflegekraft des Lieferanten wählt die entsprechenden Produkte und Mengen aus und weist die Pflegenden in die Handhabung ein.

Der Homecare-Lieferant fordert die erforderlichen Rezepte beim Arzt an. Dieser stellt die Verordnungen oftmals aus, ohne den Patienten eingehend untersucht oder auch nur gesehen zu haben. Der Arzt kann die Rezeptausstellung zwar verweigern, riskiert damit aber mögliche Versorgungslücken für den Patienten.

Die Homecare-Angestellten führen behandlungspflegerische sowie auch ärztliche Maßnahmen durch. Die Organisationsverantwortung bleibt dabei ungeklärt und die Anordnungsverantwortung des Arztes wird übergangen. 
Der Arzt verletzt damit sämtliche Anforderungen an eine geordnete Delegation. Er kommt weder seiner Auswahl-, noch Anleitungs-, noch Überwachungspflicht nach. Er kann die medizinische Notwendigkeit bzw. Leitlinienkonformität und auch fachliche Eignung des Personals nicht prüfen. In der Regel besteht auch keine schriftliche Vereinbarung über die Delegation an den Homecare-Lieferanten.

Dieses Vorgehen wird bislang von den Beteiligten geduldet. Zum Teil, weil sie bislang keinen direkten Schaden davon tragen und zum Teil, weil eine passende Lösung nicht ohne Komplikationen umgesetzt werden kann. Insbesondere Hausärzte sollten aber ein Interesse an einer Verbesserung der Situation haben. Zum einen haften sie mit der Verordnung persönlich für alle durchgeführten Maßnahmen. Zum anderen gehen sie auch ein wirtschaftliches Risiko ein, denn nur auf Grundlage der Rezeptanforderung ist der Arzt nicht in der Lage die Wirtschaftlichkeit der angeforderten Produkte zu prüfen. 